\title{
Are cardiovascular reflexes more commonly impaired in patients with bronchial carcinoma?
}

\author{
GA GOULD, M AริHWORTH, GTR LEWIS
}

From the Department of Respiratory Medicine, Ham Green Hospital and Bristol Royal Infirmary, Bristol

ABSTRACT Non-invasive tests of cardiovascular autonomic function were performed in 69 patients with histologically proved bronchial carcinoma and the results compared with those obtained in $\vec{a}$ group of age and sex matched controls. Only two patients were under 50 years of age, and with the exception of the heart rate response to deep breathing the tests performed have no accepted normat ranges in patients of this age. None of the patients had features of florid, disabling autonomico neuropathy: All the tests of autonomic function showed declining performance with age but in addition there were significant differences in the results when the two groups were compared. In the group with carcinoma the resting heart rate was higher $(\mathrm{p}<0.05)$, the resting supine blood pressure lower $(p<0.01)$, and the postural fall in blood pressure greater $(p<0.01)$. Test results were noto related to tumour histology, the presence of finger clubbing, drug history, or symptoms suggestive of autonomic dysfunction. Abnormal responses in tests of cardiovascular autonomic function are commonly found in elderly patients but bronchial carcinoma appears to have an additional effects The precise mechanism of this effect remains a matter for speculation.

Bronchial carcinoma is associated with various forms of neuropathy and myopathy. ${ }^{12}$ Autonomic dysfunction has been reported both as a consequence of malignant infiltration of nerves ${ }^{3-5}$ and as a nonmetastatic effect, ${ }^{6-13}$, although the incidence of this syndrome is unknown. The most frequently reported symptoms of autonomic neuropathy are dizziness on standing and syncope, weakness, impotence, sweating abnormalities, and bowel or bladder disturbances. $^{1415}$ Diabetic patients with autonomic neuropathy are also more prone to sudden death, perhaps related to cardiac denervation. ${ }^{16}$ If autonomic dysfunction is a complication of bronchial carcinoma it may contribute to the non-specific frailty and malaise of these patients and might also account for some of the deaths that occur without an obvious cause.

In this study we have compared the results of cardiovascular autonomic function tests in a group of patients with proved bronchial carcinoma with the results in a group of age and sex matched controls to determine whether autonomic dysfunction is

Address for reprint requests: Dr GA Gould, Rayne Laboratory, City Hospital, Edinburgh EH10 5SB.

Accepted 18 November 1985 significantly more common in patients with bronchia carcinoma.

\section{Patients and methods}

From September 1982 to January 1983 inclusive $a$ patients admitted for investigation of bronchial car cinoma were studied and the 69 with histologically. proved tumours were included in the carcinomi group (54 men, 15 women; mean age 66 years). Sixt nine age and sex matched controls $(54 \mathrm{M}, 15 \mathrm{~F}$; meas age 66 years) were studied concurrently; most of these patients were studied before elective non-thoracio surgery (for non-malignant conditions), and the rest of the group comprised non-acute medical and gerios atric inpatients and those patients with negative ref sults from investigations for bronchial carcinoman Details of age, sex, regular medication, tumour histo ogy, presence of finger clubbing, history of chronit bronchitis, and symptoms suggestive of autonomie dysfunction (postural dizziness, sweating abnormats ities, or altered bowel function) were recorded (table 1). None of the patients studied was suffering froms overt cardiac failure, chronic renal failure or diabetes mellitus and none gave a history of alcoholism.

The following tests of cardiovascular autonomi function were performed in sequence: 
Table 1 Details of the two groups of patients

\begin{tabular}{lll}
\hline & Carcinoma & Control \\
\hline Total No & $69(54 \mathrm{M}, 15 \mathrm{~F})$ & $69(54 \mathrm{M}, 15 \mathrm{~F})$ \\
Age (y) range & $43-85$ & $46-87$ \\
mean & 66 & 66 \\
Tumour histology & 38 squamous & \\
& 11 adenocarcinoma & \\
& 9 anaplastic & \\
& 9 oat cell & \\
Finger clubbing & 1 alveolar cell & 0 \\
Chronic bronchitis & 1 mesothelioma & 10 \\
Autonomic symptoms & 8 & - \\
& 2 postural dizziness & 4 diuretic \\
Medication* & 2 diarrhoea & 1 fluphenazine/nortriptyline (Motival) \\
& 1 postural dizziness and diarrhoea & \\
\hline
\end{tabular}

*Excluding benzodiazepines, non-opiate analgesics, and inhaled $\beta_{2}$ sympathomimetics.

1 The heart rate and blood pressure were measured after the patients had rested supine for at least five minutes.

2 The heart rate response to deep breathing was measured with maximal breaths at a frequency of six breaths a minute, the mean values from three consecutive breaths being used and the results being expressed as the E:I ratio-that is,

\section{longest R-R interval during expiration \\ shortest $R-R$ interval during inspiration}

3 The heart rate response to the Valsalva manoeuvre was measured during a strain of $40 \mathrm{~mm}$ $\mathrm{Hg}$ for 15 seconds, the results being expressed as the Valsalva ratio- that is,

\section{longest $R-R$ interval during recovery shortest $R-R$ interval during strain}

4 The heart rate and blood pressure response to standing was measured, the maximum fall in blood pressure being recorded and the heart rate response being expressed as the $30: 15$ ratio- that is,

longest $R-R$ interval around 30th beat after standing shortest R-R interval around 15th beat after standing

$R-R$ intervals were measured from an ECG. Blood pressure was measured to the nearest $5 \mathrm{~mm} \mathrm{Hg}$. All measurements and calculations were carried out inde- pendently by two investigators (GAG and MA) without knowledge of the patients' details.

For statistical analysis of the results we used Student's $t$ test for group data.

\section{Results}

Details of the patients are shown in table 1. From analysis of individual patient's results the presence of finger clubbing, history of chronic bronchitis, use of medication, and symptoms of autonomic dysfunction apeared to have little relationship to the results of the tests. One of the patients in the control group was taking a combined preparation of fluphenazine and nortryptiline but all of his test results were normal and he was therefore included in the analysis. None of the patients complaining of dizziness on standing had objective evidence of postural hypotension, and none of the eight patients with a postural fall in blood pressure of $30 \mathrm{~mm} \mathrm{Hg}$ or more complained of faintness or syncope. Tumour histology also appeared to be unimportant, with no obvious correlation between tumour types and test results.

Although in the control group the 30:15 ratio, E:I ratio, and Valsalva ratio declined with increasing age, age had a greater effect in the group with carcinoma. In the latter these three tests showed the same effects

Table 2 Results of autonomic function tests in carcinoma and control groups (mean (SD) with range)

\begin{tabular}{|c|c|c|c|}
\hline Test & Carcinoma & Controls & Significance* \\
\hline $\begin{array}{l}\text { Resting heart rate (beats/min) } \\
\text { Resting systolic blood pressure }(\mathrm{mm} \mathbf{H g}) \\
\text { E:I ratio } \\
\text { Valsalva ratio } \\
\text { Systolic blood pressure fall on standing }(\mathrm{mm} \mathbf{H g}) \\
30: 15 \text { ratio }\end{array}$ & $\begin{array}{c}87(20) 50-136 \\
144(24) 100-200 \\
1.19(0.16) 1.0-1.75 \\
1.33(0.28) 0.93-2.06 \\
13(16)+30 \text { to }-70 \\
1.02(0.05) 0.91-1.18\end{array}$ & $\begin{array}{l}80(13) 55-125 \\
155(23) 100-190 \\
1.21(0.14) 1.03-1.65 \\
1.38(0.27) 1.03-2.12 \\
6(10)+15 \text { to }-30 \\
1.03(0.07) 0.92-1.24\end{array}$ & $\begin{array}{l}p<0.05 \\
p<0.01 \\
\text { NS } \\
\text { NS } \\
\text { p }<0.01 \\
\text { NS }\end{array}$ \\
\hline
\end{tabular}

*Student's $t$ test. 
of age, but in addition a progressive increase in the resting heart rate and the degree of postural fall in blood pressure was observed with increasing age. In both groups low values for the 30:15 ratio (1.0 or less) were recorded so commonly as to make the test of little diagnostic value.

The results of the tests for the two groups are shown in table 2. In the group with carcinoma the mean resting heart rate was higher, the systolic blood pressure lower, and the postural fall in blood pressure greater. There were smaller, non-significant differences in the results of the other tests.

\section{Discussion}

Patients with bronchial carcinoma are often nonspecifically weak and frail for no obvious reason. If impaired cardiovascular reflexes are a contributory factor, intervention in the form of drug treatment or fluid and electrolyte replacement to normalise these reflexes may help to improve these patients' condition. Gross impairment of cardiovascular reflexes has been reported in association with bronchial carcinoma ${ }^{6-13}$ with improvement in some cases $^{812}$ after treatment for the tumour. We did not observe any cases of florid, disabling autonomic neuropathy in our study, but since the natural history and prevalence of this syndrome is unknown we might have been observing the early stages of autonomic dysfunction in some patients.

The tests performed in this study are standardised and simple to perform at the bedside, and have suggested normal ranges. ${ }^{151718}$ The results reflect a generalised response to a complex series of reflexes that depend not only on the autonomic nervous system but also on the ability of the heart and blood vessels to react appropriately. In young subjects we may assume that abnormalities in the responses to these tests nearly always represent autonomic damage; but in the elderly, when multiple pathological conditions are more likely, results must be interpreted with care since normal ranges are more difficult to define ${ }^{1920}$-absent or severely impaired cardiovascular reflexes may occur commonly, as is suggested by our results for the response of the heart rate response to standing (30:15 ratio). Some workers have observed little effect of age on the 30:15 ratio ${ }^{18}$ or the Valsalva ratio, ${ }^{2122}$ but very few subjects over 65 years were included in these studies. In studies that have included geriatric patients other workers have shown that the Valsalva ratio ${ }^{23}$ and the E:I ratio ${ }^{17}$ progressively decline with age and that large postural falls in blood pressure are commonly found in the elderly. ${ }^{24} 25$

Our results have confirmed the effect of age on cardiovascular reflexes, but in addition we have observed significant differences between the group with carcinoma and the control group. The other variables recorded had no obvious relationship to abnormals tests results; in the case of finger clubbing this was ano interesting observation since clubbing and hyper- $\underline{\underline{T}}$ trophic osteoarthropathy may have some connection $\frac{\bar{c}}{\sqrt{2}}$ with autonomic dysfunction, as the condition may be $\stackrel{\mathbb{Q}}{\mathbb{Q}}$ reversed by vagotomy. Although previous workerso have suggested that non-metastatic complications of bronchial carcinoma (neurological and biochemical) $\stackrel{\circ}{-}$ are more common with oat cell tumours ${ }^{12}$ we found $\vec{\omega}$ no association with a tumour type. Our numbers areo probably too small to draw any conclusions about $\vec{x}$ this.

Our findings suggest that in patients with bronchial carcinoma tests of cardiovascular function more com- $\dot{\omega}$ monly reveal impairment, but the cause of this is un-N certain. The most significant differences observed in음 our carcinoma group (higher resting heart rate, lowersupine blood pressure, greater postural fall in blood? pressure) are non-specific markers of abnormal car diovascular function and, even though the more $\vec{\bullet}$ specific tests of autonomic function (Valsalva ratio,,$\infty$ E:I ratio) also showed minor and non-significant differences, the abnormalities may not indicate auto-o nomic neuropathy but may be due to other factors. Disturbance of fluid and electrolyte balance is a likelyo cause, as many of these patients will have a poor fluido intake owing to anorexia and some will also be febrile, both of which may cause dehydration. A fewo $\overrightarrow{\overrightarrow{0}}$ patients will have inappropriate hormone secretion, which will also affect fluid and electrolyte balance and may disturb cardiovascular reflexes. Another factorthat may be important is coexisting abnormalities of pulmonary function, since some patients will have lung collapse or consolidation due to local tumourx effects, and many will have chronic airflow obstruc- -3 tion owing to their heavy smoking. The effects of $a b-$. normal pulmonary function on these cardiovascular reflexes have not been studied in a large group of patients, though one study showed impairment of the Valsalva response in three patients with em? physema ${ }^{26}$ while another study found a normal Val salva response in 10 patients with chronic pulmonary disease and no clinical evidence of right ventricular failure. ${ }^{22}$ The severity of lung disease was not docu mented in either of these studies; in our patients $x^{w}$ history of chronic bronchitis had no obvious effect on the results of the tests.

In summary, in a group of 69 elderly patients with bronchial carcinoma and 69 age and sex matched controls we have confirmed the finding that certain cardiovascular reflexes decline with age, and showe of that some tests, such as the 30:15 ratio, may be of little diagnostic value since low values occur verye commonly. We have also observed an effect of brone 
chial carcinoma, with significant differences in the mean values for the resting heart rate, supine blood pressure, and postural fall in blood pressure when the two groups are compared, although we encountered no patients with florid, disabling autonomic neuropathy of the sort previously reported. Other variables, such as tumour histology, regular medication, and symptoms suggestive of autonomic dysfunction, did not appear to influence test results. The cause of these observed differences is not apparent.

\section{References}

1 Dayan AD, Croft PB, Wilkinson M. Association of carcinomatous neuromyopathy with different histological types of carcinoma of the lung. Brain 1965;88:435-48.

2 Croft PB, Wilkinson M. The incidence of carcinomatous neuromyopathy in patients with various types of carcinoma. Brain 1965;88:427-34.

3 Kirks DR, Szemes GC. Autovagotomy and gastric bezoar. Gastroenterology 1971;61:96-8.

4 Allan TNK, Willson RA, Lee M. Bronchial carcinoma with autovagotomy and bezoar formation. JAMA 1977;237:364-5.

5 Thomas WEG, Fletcher MS. Neoplastic autovagotomy causing gastric stasis. Postgrad Med J 1979;55:411-6.

6 Ivy HK. Renal sodium loss and bronchogenic carcinoma. Associated autonomic neuropathy. Arch Intern Med 1961;108:47-55.

7 Siemsen JK, Meister L. Bronchogenic carcinoma associated with severe orthostatic hypotension. Ann Intern Med 1963;58:669-76.

8 Park DM, Johnson RM, Crean GP, Robinson JF. Orthostatic hypotension in bronchial carcinoma. $\mathrm{Br} \mathrm{Med} \mathrm{J}$ 1972;iii:510-1.

9 Ahmed MN, Carpenter S. Autonomic neuropathy and carcinoma of the lung. Can Med Assoc J 1975;113:410-2.

10 Quinlan CD. Autonomic neuropathy in carcinoma of the lung. J Irish Med Assoc 1971;84:430-1.

11 Chiappa KH, Young RR. A case of paracarcinomatous pandysautonomia. Neurology 1973;23:423.

12 Vasudevan CP, Suppiah P, Udoshi MB, Lusius J. Re- versible autonomic neuropathy and hypertrophic osteoarthropathy in a patient with bronchogenic carcinoma. Chest 1981;79:479-81.

13 Carr-Locke DL. Autonomic neuropathy and inappropriate secretion of antidiuretic hormone. Occurrence in a patient with bronchogenic carcinoma. JAMA 1979; 241:2298.

14 Hines S, Houston M, Robertson D. The clinical spectrum of autonomic dysfunction. Am J Med 1981; 70:1091-6.

15 Ewing DJ, Clarke BF. Diagnosis and management of diabetic autonomic neuropathy. $B r \operatorname{Med} J$ 1982; 285:916-8.

16 Page M McB, Watkins PJ. Cardiorespiratory arrest and diabetic autonomic neuropathy. Lancet 1978;i:14-6.

17 Smith SA. Reduced sinus arrhythmia in diabetic autonomic neuropathy: diagnostic value of an age related normal range. Br Med J 1982;285:1599-601.

18 Ewing DJ, Campbell IW, Murray A, Neilson JMM, Clarke BF. Immediate heart-rate response to standing: simple test for autonomic neuropathy in diabetes. $\mathrm{Br}$ Med J 1978;i;145-7.

19 Collins KJ, Exton-Smith AN, James MH, Oliver DJ. Functional changes in autonomic nervous responses with ageing. Age Ageing 1980;9:17-24.

20 Vargas E, Lye M. The assessment of autonomic function in the elderly. Age Ageing 1980;9:210-4.

21 Baldwa VS, Ewing DJ. Heart rate response to Valsalva manoeuvre. Reproducibility in normals, and relation to variation in resting heart rate in diabetics. Br Heart $J$ 1977;39:641-4.

22 Levin AB. A simple test of cardiac function based upon the heart rate changes induced by the Valsalva manoeuvre. Am J Cardiol 1966;18:90-9.

23 Smith SE, Smith SA. Heart rate variability in healthy subjects measured with a bedside computer-based technique. Clin Sci 1981;61:379-83.

24 Caird FI, Andrews GR, Kennedy RD. Effect of posture on blood pressure in the elderly. Br Heart J 1973; 35:527-30.

25 Johnson RH, Smith AC, Spalding JMK, Wollner L. Effect of posture on blood pressure in elderly patients. Lancet 1965;i:731-3.

26 Cudcowicz L. Normal, pharmacological and disordered circulatory responses to the Valsalva manoeuvre. Respiration 1968;25:81-115. 